\title{
Casuística de um Serviço de Urgência: Motivos de Referenciação dos Cuidados de Saúde Primários
}

\author{
Casuistry of an Emergency Service: Reasons for Referencing Primary \\ Health Care
}

\begin{abstract}
Inês Vieira Santos (https://orcid.org/0000-0002-2256-0119), Susana Escária (https://orcid.org/ 0000-0003-2930-297X), Maria Piteira (https://orcid.org/0000-0001-9355-4923), Sofia Calaça (https://orcid.org/0000-0002-3713-1472), Diogo Canudo (https://orcid.org/0000-0002-7872-0652), Alicia Oliveira (https://orcid.org/0000-0003-0833-0736), Claudiu Guz (https://orcid. org/0000-0002-7465-8070), Ireneia Lino (https://orcid.org/0000-0002-2346-6714)
\end{abstract}

\section{Resumo:}

Introdução: São referenciados diariamente um elevado número de doentes, provenientes dos cuidados de saúde primários, ao serviço de urgência hospitalar. Este trabalho tem como objectivo identificar os principais motivos de referenciação, a abordagem no serviço de urgência e o destino destes doentes.

Material e Métodos: Estudo descritivo. Foram seleccionados todos os doentes provenientes dos cuidados de saúde primários (CSP), localizados na ARS Alentejo Central, referenciados ao serviço de urgência (SU) do Hospital Espírito Santo de Évora, triados para a especialidade de Medicina interna, no mês de Janeiro e Julho de 2017. Para realização da análise estatística utilizou-se o Speed Statistics ${ }^{\circledR}$ (SPSS).

Resultados: Aproximadamente 15\% dos doentes admitidos no serviço de urgência são provenientes dos CSP e 50\% destes são triados para a Medicina interna. Cerca de 57\% dos doentes apresentam prioridade urgente e o motivo mais frequente de referenciação é a sintomatologia respiratória. Uma grande percentagem realiza exames complementares de diagnóstico. Verificou-se que $75 \%$ dos doentes admitidos tiveram alta para o domicílio, sendo que 25\% não apresentava patologia aguda ou não foi feito diagnóstico no SU.

Discussão e Conclusão: São necessários mais estudos para identificar quais as lacunas no percurso do doente com patologia aguda e o que motiva a transferência dos doentes dos CSP ao SU. É preciso reflectir e mudar a estratégia, com o objectivo de melhorar a eficiência do sistema nacional de saúde, culminando num maior conforto para o doente. Seria ainda aliciante a formação de equipas multidisciplinares, de apoio aos CSP, para discussão de casos de patologia aguda e decisão de qual a melhor abordagem diagnóstica e terapêutica. Desta forma seria possível diminuir o consumo de recursos, criando uma abordagem mais centrada no doente.

Palavras-chave: Cuidados Saúde Primários; Doença Aguda; Serviço de Urgência Hospitalar.

Serviço de Medicina Interna e Serviço de Urgência, Hospital do Espírito Santo de Évora, Évora, Portugal

DOI:10.24950/O/224/20/1/2021

\section{Abstract:}

Introduction: A large number of patients from primary health care are referred to the hospital emergency department daily. The aim of our study is to identify the major reasons for that, the approach in the emergency department and the destination of these patients.

Material and Methods: Descriptive study. All patients from primary health care (PHC) were selected at ARS Alentejo Central, referred to the emergency department (ED) of Hospital Espírito Santo de Évora, for the specialty of Internal Medicine, in the month of January and July 2017. To perform the statistical analysis, the Speed Statistics $®$ (SPSS) was used.

Results: About $15 \%$ of patients admitted to the emergency department are from PHC and 50\% of these are forwarded for internal medicine. About $57 \%$ of patients have priority and the most frequent reason for referral is respiratory symptoms. A major conclusion carries out complementary diagnostic tests. It was found that $75 \%$ of the admitted patients were discharged to the home, $25 \%$ of whom had no acute pathology or had not been diagnosed in the ED.

Discussion and Conclusion: More studies are necessary to identify the gaps in the path of the patient with acute pathology and what motivates the transfer of patients from PHC to ED. It is necessary to reflect and change the strategy, with the objective of improving the efficiency of the national health system, culminating in greater comfort for the patient. It would also be attractive to make multidisciplinary teams, in support of PHC, to discuss cases of acute pathology and decide on the best diagnostic and therapeutic approach. In this way, it would be possible to reduce the consumption of resources, creating a more patient-centred approach.

Keywords: Acute Disease; Emergency Service, Hospital; Primary Health Care.

\section{Introdução}

Os serviços de urgência hospitalar estão frequentemente lotados..$^{1,2}$ Este uso excessivo implica elevados custos, mas também tempos de espera prolongados e, inevitavelmente, um pior atendimento do doente. O doente tem 
autonomia para escolher onde quer ser observado, ou nos cuidados de saúde primários ou em meio hospitalar. ${ }^{3}$

No entanto, verifica-se que são referenciados diariamente um elevado número de doentes, provenientes dos cuidados de saúde primários, para o serviço de urgência hospitalar.

Este trabalho tem como objectivo identificar os principais motivos de referenciação ao serviço de urgência e qual o destino do doente referenciado.

\section{Material e Métodos}

Estudo descritivo. Foram seleccionados todos os doentes provenientes dos cuidados de saúde primários, localizados na ARS Alentejo Central, referenciados ao serviço de urgência (SU) do Hospital Espírito Santo de Évora, no mês de Janeiro e Julho de 2017.

Para a análise estatística, foram incluídos apenas os doentes triados para a especialidade de Medicina Interna. Foram excluídos doentes em idade pediátrica ( $n=18)$, referenciados para a Cirurgia Geral $(n=428)$, Ortopedia ( $n=$ 320), Oftalmologia ( $n=144)$, Ginecologia/Obstetrícia $(n=64)$, Otorrinolaringologia $(n=10)$, Psiquiatria $(n=9)$ e Cardiologia $(n=2)$ (Fig. 1).

Foram avaliadas as variáveis: idade, sexo, motivo de referenciação e sintomatologia, prioridade clínica, realização de meios complementares de diagnóstico (MCD) e de terapêutica, tempo de permanência no SU (tempo total desde a admissão), destino e diagnóstico à data da alta.

Para caracterizar o motivo de referenciação foram criadas as variáveis "sintomatologia respiratória", "sintomatologia abdominal" e "sintomatologia urinária" e "sintomas constitucionais". Por não se enquadrarem em nenhuma das variáveis previamente descritas foram criadas as variáveis "palpitações/dor torácica", "défice neurológico focal, alteração do estado de consciência (AEC) e convulsões", "cefaleia/tonturas/crise hipertensiva não controlada", "lipotímia/ síncope", "dor osteomuscular", "alterações analíticas", "odinofagia/otalgia", "intoxicação medicamentosa voluntária (IMV)/ descompensação de patologia psiquiátrica", "alterações cutâneas/reacção alérgica", "edema dos membros inferiores" e por fim "outros" (sintomas que não se incluem em nenhuma das variáveis descritas anteriormente).

Foi realizada uma análise descritiva dos dados, em que a determinação da normalidade das variáveis contínuas foi obtida através da medida de assimetria e curtose. Nas variáveis com distribuição normal avaliou-se a média e o desvio padrão; nas variáveis sem distribuição normal foi avaliada a mediana e amplitude interquartil.

Foi avaliado o valor mínimo e máximo da variável idade. As variáveis categóricas são representadas pelo seu valor absoluto (n) e respectiva percentagem (\%).

Para realização da análise estatística utilizou-se o Speed Statistics ${ }^{\circledR}$ (SPSS).

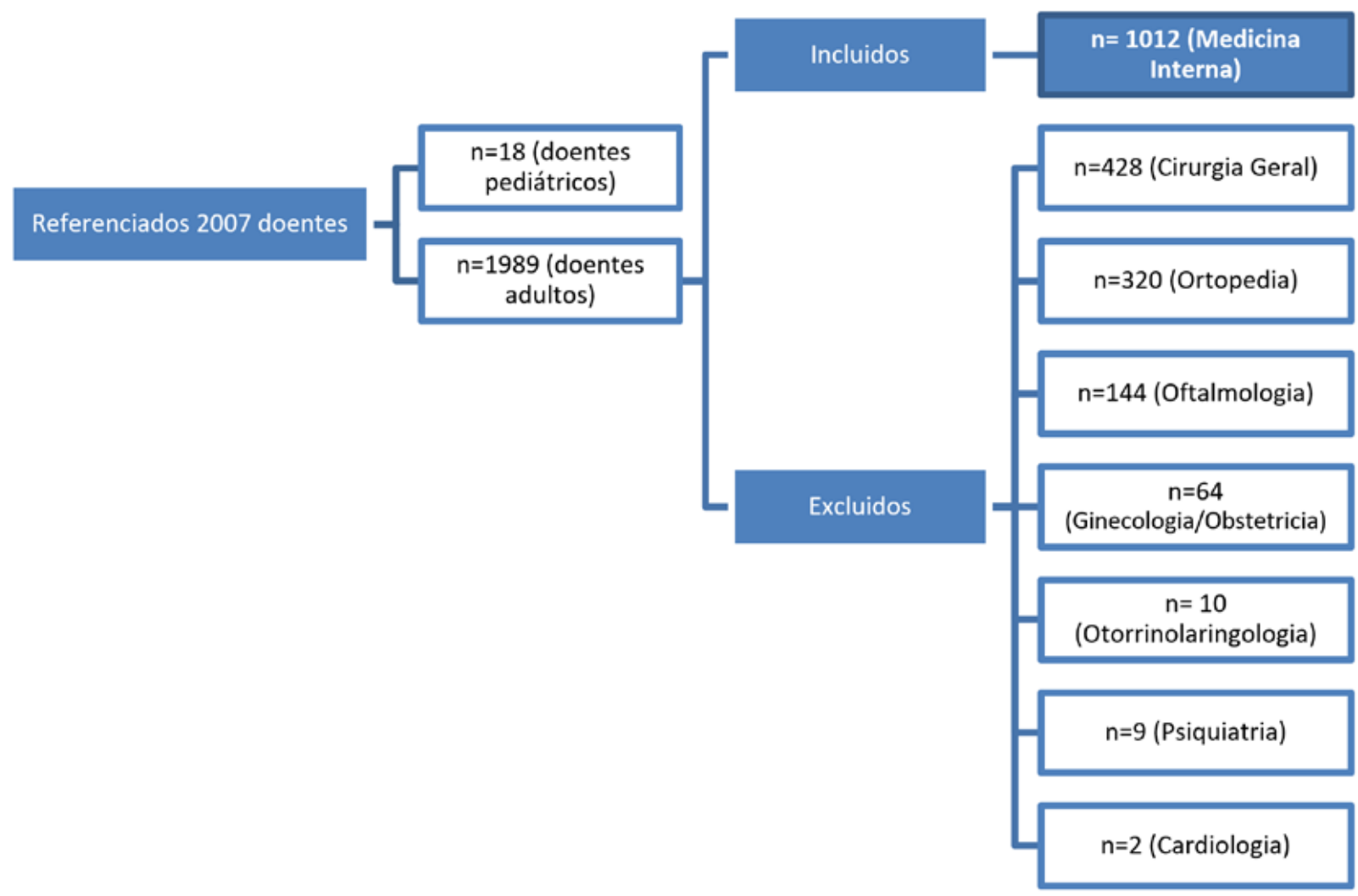

Figura 1: Método de selecção dos doentes para análise estatística. 


\section{Resultados}

No mês de Janeiro e Julho de 2017 foram admitidos no serviço de urgência um total de 12,526 doentes e destes, $55,5 \%$ ( $n=6,951$ ) foram triados para a especialidade de medicina interna.

Foram referenciados 1989 doentes dos centros de saúde da ARS Alentejo Central para o serviço de urgência hospitalar. No mês de Janeiro e Julho foram admitidos 1502 e 937 doentes, respectivamente. Este valor corresponde a $15,9 \%$ do total de doentes admitidos no serviço de urgência.

Destes doentes, cerca de 50,9\% ( $n=1012)$ foram triados para a especialidade de Medicina interna.

\section{SEXO E IDADES}

Aproximadamente 55,3\% ( $n=560)$ dos doentes eram do sexo feminino e $44,7 \%(n=452)$ do sexo masculino. A média de idades foi de 67,6 anos ( $\pm 19,7)$; sendo o mínimo 18 anos e o máximo 101 anos. A maioria dos doentes tem idade superior ou igual a 80 anos.

\section{PRIORIDADE CLÍNICA}

Como é possível verificar, a maioria dos doentes, foram triados com prioridade clínica "Urgente - Amarelo", correspondendo a 56,7\% ( $n=574)$ dos doentes; em 31,6\% ( $n=$ 320) foi atribuída a prioridade clínica "Muito urgente - Laranja"; em 9,8\% ( $n=99$ ) prioridade "Pouco urgente - Verde"; em $1,5 \%(n=15)$ prioridade "Não urgente - Azul" e por fim, $0,4 \%$ dos doentes $(n=4)$ apresentaram prioridade clínica "Emergente - Vermelho" (Fig. 2)

\section{MOTIVOS DE REFERENCIAÇÃO}

Verificou-se que o motivo mais frequente de referenciação (Fig. 3) foi a sintomatologia respiratória, em 20,1\% ( $n=203$ ) dos doentes.

De seguida, a dor torácica ou palpitações, em 13,2\% ( $n=$ 134). Em 12,3\% ( $n=124)$ o motivo de referenciação foi sintomatologia abdominal; e em 9,8\% ( $n=99)$ apresentavam sintomas constitucionais. Em igual percentagem, em 8,7\% ( $n=88)$ foram enviados doentes por défice neurológico focal, convulsões ou alteração do estado de consciência (AEC), bem como por cefaleias, tonturas ou crise hipertensiva não controlada. Foram ainda referenciados 5,9\% $(n=60)$ doentes por sintomatologia urinária ou dor lombar; aproximadamente 4,2\% ( $n=43)$ foram referenciados por dor osteomuscular; e por fim em 3,5\% ( $n=$ 35) por alterações cutâneas ou reacção alérgica. Foram referenciados ainda alguns doentes com sintomatologia que não se enquadrava em nenhum motivo de referenciação especificado anteriormente (3,2\%, $n=32)$; por alterações analíticas $(2,8 \%, n$ $=28$ ), doentes com queixas de otalgia, odinofagia ou odontalgia $(2,3 \%, n=23)$ e ainda por intoxicação medicamentosa voluntária (IMV) ou descompensação de patologia psiquiátrica $(2,3 \%, n$ = 23). Foram referenciados doentes com episódio de lipotimia ou síncope $(2,1 \%, n=21)$; e por fim por epistáxis não controlada nos cuidados de saúde primários $(1,1 \%, n=11)$.

\section{EXAMES COMPLEMENTARES DE DIAGNÓSTICO E TERA- PÊUTICA}

Verificou-se que 90,6\% ( $n=917$ ) dos doentes realizou exames complementares de diagnóstico e em 73,2\% ( $n=741)$ dos doentes foi prescrito terapêutica.

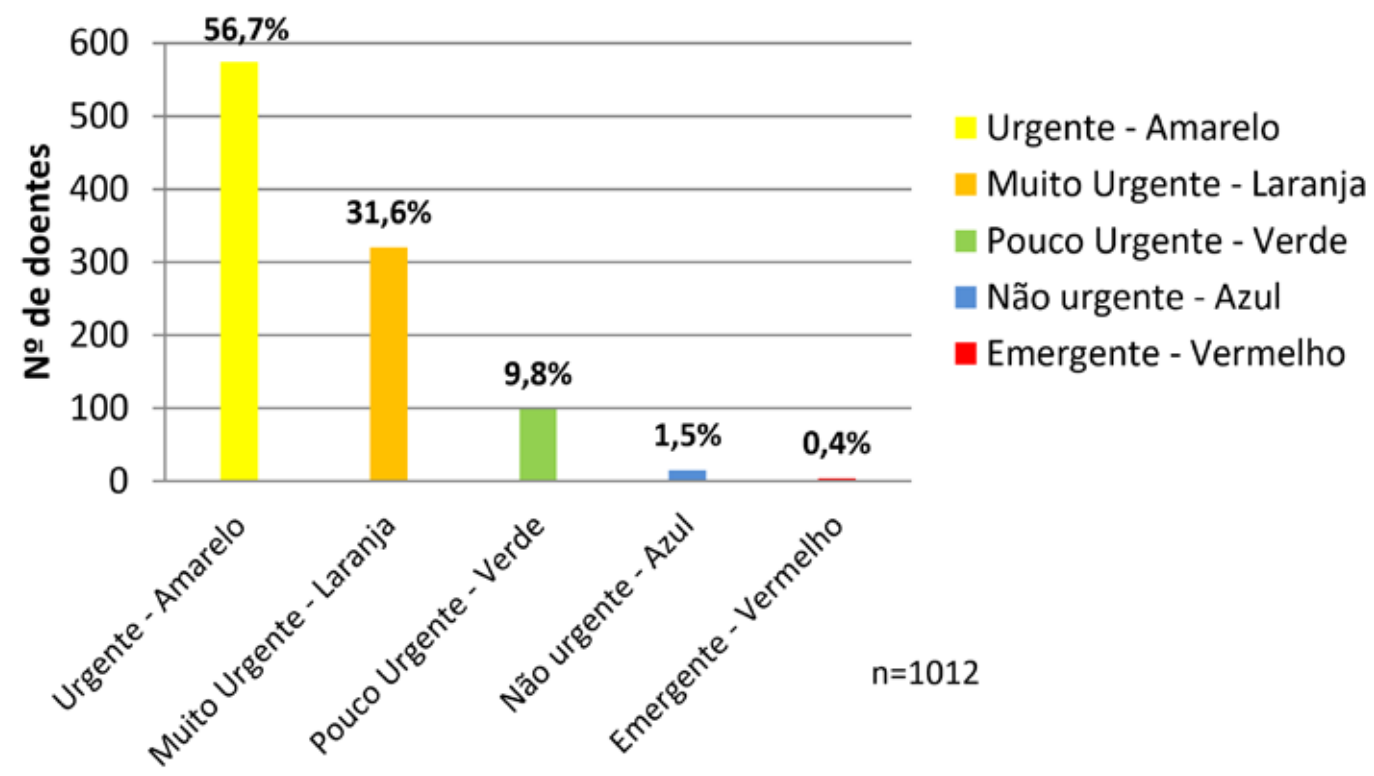

Prioridade Clínica

Figuira 2: Prioridade clínica dos doentes referenciados dos cuidados de saúde primários. 


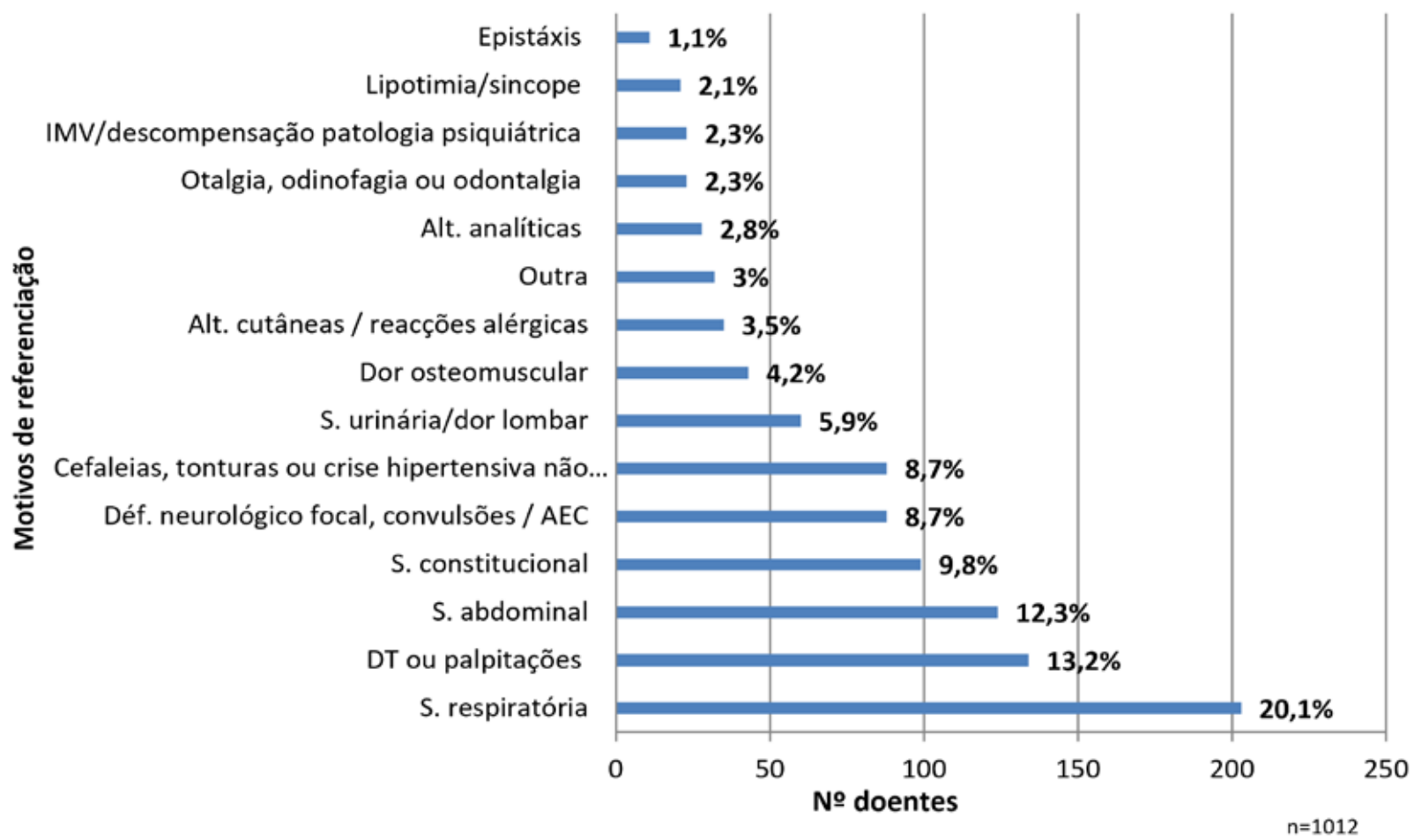

Figura 3: Motivos de referenciação dos doentes provenientes cuidados de saúde primários.

\section{TEMPO DE PERMANÊNCIA NO SERVIÇO DE URGÊNCIA E DESTINO}

Foi avaliado o tempo de permanência desde a admissão no serviço de urgência. Verificou-se que a mediana foi de 6 horas, com uma amplitude interquartil de 9 horas, sendo o mínimo inferior a 1 hora e o máximo de 72 horas.

Analisando o destino destes doentes Fig. 4), verificou-se que $75 \%$ dos doentes $(n=759)$ teve alta para o exterior $e$ $17,6 \%(n=178)$ foram internados. Cerca de 3,2\% ( $n=32)$ foram referenciados para uma consulta externa hospitalar e em 1,8\% ( $n=18)$ dos casos houve necessidade de transferência para outro hospital, para apoio diferenciado. Cerca de $1,1 \%(n=11)$ dos doentes faleceram; $0,9 \%(n=9)$ dos doentes abandonou o serviço de urgência e por fim, 0,5\% ( $n=5)$ dos doentes pediram alta contra parecer médico.

\section{DIAGNÓSTICO À DATA DE ALTA NOS DOENTES COM ALTA PARA O EXTERIOR}

Como referido anteriormente, cerca de 75\% ( $n=759$ ) dos doentes tiveram alta para o exterior. Avaliou-se o diagnóstico à data da alta destes doentes (Fig. 5) e verificou-se que $25,3 \%$ ( $n=192$ ) destes teve alta sem diagnóstico, ou por não apresentar patologia aguda.

Entre os diagnósticos mais frequentes encontra-se a infecção respiratória baixa não especificada em 9,6\% ( $n=$ 73); a cistite em 5,3\% ( $n=40)$; a insuficiência cardíaca descompensada em 5,1\% $(n=39)$ dos doentes. Cerca de $3 \%$
( $n=23)$ dos doentes teve diagnóstico à data da alta de dorsopatia não especificada; em 2,8\% ( $n=21)$ de crise/urgência hipertensiva, e em 2,2\% ( $n=17)$ de anemia.

Em igual percentagem, em 2,2\% ( $n=17)$ o doente teve diagnóstico de gastroenterite aguda ou colite a agente desconhecido, síndrome vertiginoso ou fibrilhação auricular (FA) / flutter auricular.

Em 2,1\% ( $n=16)$ distúrbio de ansiedade não especificado e em 1,8\% $(n=14)$ diagnóstico de doença do refluxo gastroesofágico (DRGE). Em menor percentagem, diagnosticaram-se doentes com reacção alérgica, cólica renal, amigdalite, otite externa não especificada, erisipela, obstipação, artrose não especificada e síndrome de dependência alcoólica.

\section{Discussão e Conclusão}

Todos os dias são referenciados inúmeros doentes ao serviço de urgência hospitalar provenientes nos cuidados de saúde primários. A maioria destes são doentes idosos, com idade superior ou igual a 80 anos e os que mais recursos consomem na saúde. ${ }^{5}$ Verificou-se que embora tenha sido atribuída prioridade urgente a mais de metade dos doentes, aproximadamente $75 \%$ da totalidade destes, teve alta para o exterior. Questiona-se assim, se houve efectivamente benefício para o doente, em deslocar-se a um serviço de urgência hospitalar, por vezes com serviços sobrelotados, com maior tempo de espera e com um atendimento menos 


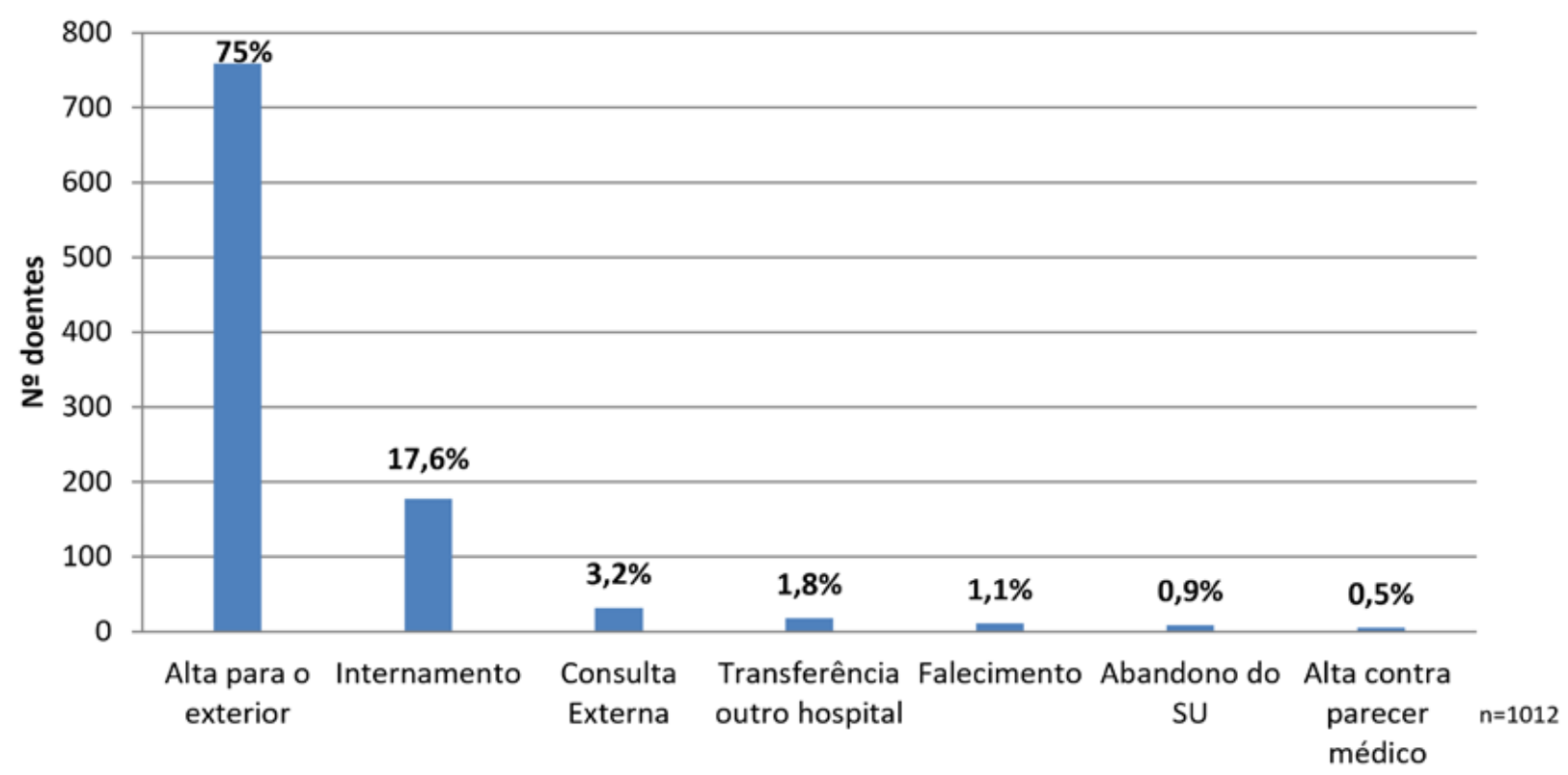

Destino dos doentes

Figura 4: Destino dos doentes provenientes cuidados de saúde primários.

dirigido, por um médico que não conhece o doente e que não vai avaliar a evolução clínica do mesmo, a quem prescreveu uma determinada terapêutica. Em cerca de 25\% dos doentes que teve alta para o exterior, não foi identificada patologia aguda nem realizado nenhum diagnóstico, no serviço de urgência. Possivelmente, estes doentes poderiam ter sido orientados pelo seu médico assistente, no âmbito dos cuidados de saúde primários, sem necessidade de recorrer ao serviço de urgência. Numa elevada percentagem, em cerca de $90 \%$ dos doentes, foram realizados exames complementares de diagnóstico. Por um lado, a necessidade de realização de exames complementares em tempo

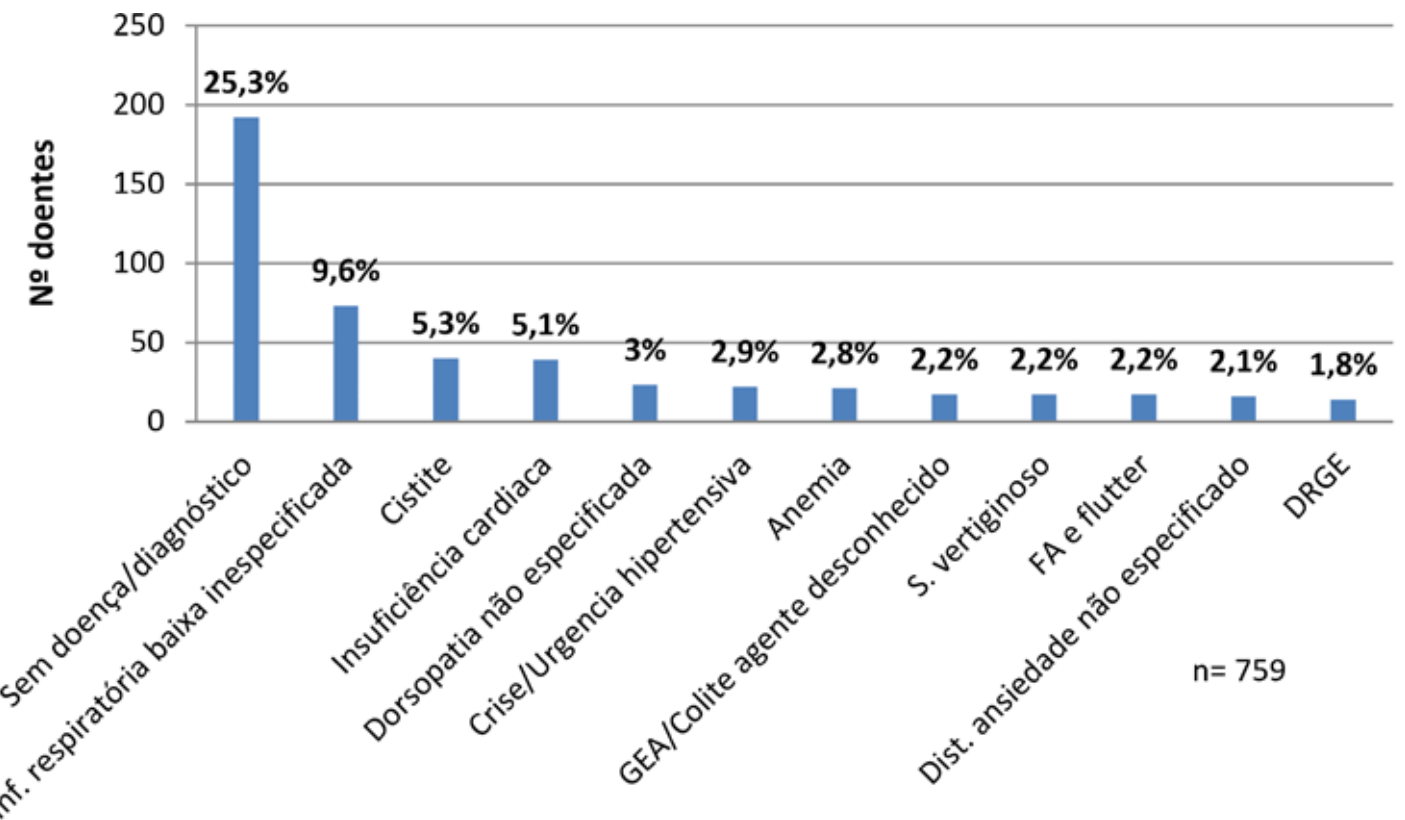

Diagnósticos

GEA - gastrenterite aguda; FA - Fibrilhação auricular; DRGE - Doença refluxo gastro-esofágico

Figura 5: Diagnóstico dos doentes com alta para o exterior. 
útil pode justificar a necessidade de transferência para um serviço de urgência hospitalar, no entanto, cada vez mais exerce-se uma medicina defensiva e consequentemente são prescritos mais exames. ${ }^{4}$

Constatou-se que foi prescrita terapêutica a $73 \%$ dos doentes, onde se inclui maioritariamente terapêutica oral como analgésicos e antipiréticos, fluidoterapia e terapêutica antimicrobiana.

São necessários mais estudos para perceber quais as lacunas no percurso do doente com patologia aguda e o que motiva a transferência dos doentes dos CSP ao SU. É preciso reflectir e mudar a estratégia, com o objectivo de melhorar a eficiência do sistema nacional de saúde, culminando num maior conforto para o doente. Seria benéfico se alguns CSP fossem dotados de meios de diagnóstico para que fossem autónomos no diagnóstico de patologias com menor gravidade clínica. Seria ainda aliciante a formação de equipas multidisciplinares, de apoio aos CSP, para discussão de casos de patologia aguda e decisão de qual a melhor abordagem diagnóstica e terapêutica. Para isso é necessário melhorar a comunicação entre os diferentes níveis de cuidados. Desta forma seria possível diminuir o consumo de recursos, criando uma abordagem mais centrada no doente. ${ }^{1}$

\section{Responsabilidades Éticas}

Conflitos de Interesse: Os autores declaram a inexistência de conflitos de interesse na realização do presente trabalho.

Fontes de Financiamento: Não existiram fontes externas de financiamento para a realização deste artigo

Confidencialidade dos Dados: Os autores declaram ter seguido os protocolos da sua instituição acerca da publicação dos dados de doentes.

Proteção de Pessoas e Animais: Os autores declaram que os procedimentos seguidos estavam de acordo com os regulamentos estabelecidos pelos responsáveis da Comissão de Investigação Clínica e Ética e de acordo com a Declaração de Helsínquia da Associação Médica Mundial. Proveniência e Revisão por Pares: Não comissionado; revisão externa por pares.

\section{Ethical Disclosures}

Conflicts of interest: The authors have no conflicts of interest to declare. Financing Support: This work has not received any contribution, grant or scholarship

Confidentiality of Data: The authors declare that they have followed the protocols of their work center on the publication of data from patients.

Protection of Human and Animal Subjects: The authors declare that the procedures followed were in accordance with the regulations of the relevant clinical research ethics committee and with those of the Code of Ethics of the World Medical Association (Declaration of Helsinki).

Provenance and Peer Review: Not commissioned; externally peer reviewed.

(C) Autor (es) (ou seu (s) empregador (es)) e Revista SPMI 2021. Reutilização permitida de acordo com CC BY-NC. Nenhuma reutilização comercial. (c) Author(s) (or their employer(s)) and SPMl Journal 2021. Re-use permitted under CC BY-NC. No commercial re-use.

\section{Correspondence / Correspondência:}

Inês Vieira Santos - inesvieira.santos@gmail.com

Serviço de Medicina Interna e Serviço de Urgência, Hospital do Espírito Santo de Évora, Évora, Portugal

Largo do Sr. da Pobreza, 7000-811 Évora

Received / Recebido: 26/10/2020

Accepted / Aceite: 22/01/2021

Publicado / Published: 15 de março de 2021

\section{REFERÊNCIAS}

1. Saúde em análise, Uma visão para o futuro. Lisboa: Deloitte; Public Sector, Life Sciences \& Healthcare; 2011.

2. Brito e Sá A. Urgência hospitalar e Cuidados de Saúde Primários: mitos e falácias. Rev Port Clin Geral. 2002;18:347-8

3. Joaquim Palma; Problemas de Saúde Urgentes: a quem Recorrem os Utentes e o que Influencia a sua Escolha; Rev Port Clin Geral. 2002;18:28397

4. Rosas de Casto J. Consentimento informado e medicina defensiva. JULGAR; 2014

5. Observatório Português dos Sistemas de Saúde; Meio caminho andado; Relatório primavera 2018. Lisboa: OPSS; 2018. 